\title{
Comment on: development and external validation of a model to predict overall survival in patients with resected gallbladder cancer
}

\author{
Sakti Chakrabarti ${ }^{1}$, Amit Mahipal ${ }^{2}$ \\ ${ }^{1}$ Medical College of Wisconsin, Milwaukee, WI, USA; ${ }^{2}$ Division of Medical Oncology, Mayo Clinic, Rochester, MN, USA \\ Correspondence to: Amit Mahipal, MBBS, MPH. Department of Oncology, Mayo Clinic, 200 1st Street SW, Rochester, MN 55902, USA. \\ Email: Mahipal.amit@mayo.edu. \\ Comment on: Lohman EAJS, de Bitter TJJ, Hannink G, et al. Development and External Validation of a Model to Predict Overall Survival in Patients \\ with Resected Gallbladder Cancer. Ann Surg 2021. [Epub ahead of print]. doi:10.1097/SLA.0000000000005154.
}

Submitted Dec 03, 2021. Accepted for publication Dec 13, 2021.

doi: $10.21037 / \mathrm{hbsn}-21-514$

View this article at: https://dx.doi.org/10.21037/hbsn-21-514

We read the study reported by Lohman et al. with interest published recently in the Annals of Surgery (1). The authors have developed a statistical model to predict the overall survival (OS) of patients with resected gall bladder cancer (GBC). Dutch national database was used to create and internally validate the model based on individual-level data $(\mathrm{n}=380)$. Subsequently, data from a cohort of Australian patients with resected GBC were used for external validation $(\mathrm{n}=66)$. The model was constructed based on the patient and tumor characteristics found to be independent predictors of OS that included age, tumor and node $(\mathrm{T} / \mathrm{N})$ classification, resection margin, tumor differentiation grade, and vascular invasion. The concordance index $(\mathrm{C}$-index $)$ in the internal and the external validation cohorts were 0.71 [95\% confidence interval (CI): 0.69-0.72] and 0.75 (95\% CI: 0.69-0.80), respectively, suggesting good discriminatory capacity. The authors also demonstrated that their model outperformed the discriminative capability of the American Joint Committee on Cancer (AJCC) staging system (8th edition) with a C-index of 0.59 (95\% CI: $0.57-0.60$ ). Furthermore, the authors constructed a web application that can predict the OS of a given patient based on a small number of clinicopathologic variables (age, $\mathrm{T}$ stage, $\mathrm{N}$ stage, resection margin, differentiation grade, and perivascular invasion).

Although a rare neoplasm in general, GBC is the most common malignant tumor originating from the biliary tree with a wide geographic variation in annual incidences, ranging from 2 per 100,000 people in the United States to 27 per 100,000 people in Chile (2). GBC is an aggressive malignancy with a poor prognosis and a 5 -year OS rate of only $12 \%$ (3). Surgical resection is the only curative treatment for patients with non-metastatic disease, and radical resection has been shown to improve survival (3). Although most patients with GBC present with unresectable advanced disease, the presentation pattern is different in Western countries than in the rest of the world. In western countries, GBC is diagnosed incidentally after cholecystectomy for presumed benign pathology, and often these patients do not harbor metastatic disease detectable by imaging studies allowing curative-intent treatment (4). In patients with incidentally diagnosed GBC, re-resection is usually considered to eradicate residual cancer and perform a complete staging lymphadenectomy (4). However, a well-agreed standard treatment protocol for such patients does not exist. A risk-stratification tool in such patients can be of much value to guide the decision of re-resection, design adjuvant clinical trials with novel therapies, compare the outcome with different treatment protocols, and inform patients of their prognosis. The most widely used prognostic tool is the AJCC staging system, which has a modest predictive capability, as demonstrated in many studies (5-7). Several prognostic models for patients with GBC have been reported thus far, both in resected (8-13) and advanced (12) patient population. With the exception of a large study by Yadav et al., majority of the prognostic models in resected patients have been constructed based on data from non-western patients (12).

The model reported by Lohman and colleagues has 
several unique and valuable characteristics. First, the model was constructed based on a database that contained individual-level data on a large group of western patients. Most prediction models published thus far included either non-western patients or patients from a large database lacking detailed individual-level clinicopathologic data. Second, the histopathology of all patients included in the development and the validation cohorts, including the external validation cohort, were reviewed by the same group of expert pathologists to ensure data quality. This enormous effort deserves applause. Third, most patients $(77.5 \%)$ in the development cohort were diagnosed incidentally after cholecystectomy for a presumed benign pathology that reflects the western pattern of presentation, ensuring the applicability of the model to the western patients. Fourth, the authors validated the model in an external dataset that showed a high c-index of 0.75 . Finally, the proposed model outperformed the AJCC staging system in predicting survival. Furthermore, the web application derived from this model provides a simple and easy way of predicting prognosis in resected GBC patients that can be quite useful in routine clinical practice. This study indeed provides a robust reference for the western patients.

Several aspects of this study, however, need a critical appraisal. Among the prognostic factors in patients with resected GBC, lymph node (LN) metastasis has been demonstrated to impact the OS significantly $(10,14)$. In the model development cohort of the current study, most patients $(59.7 \%)$ did not have any information regarding the LN status (NX) that limited the accuracy of the model. Furthermore, the 8th edition of the AJCC staging system recommends at least $6 \mathrm{LN}$ examinations in the pathology specimen for accurate staging. The authors did not provide any information regarding the number of $\mathrm{LN}$ examined in the pathology specimens. Lack of $\mathrm{LN}$ assessment limits the comparison of this model to the AJCC staging system. As with the AJCC system, the new model requires data from the pathologic examination of the resected specimen, limiting the applicability of this model to patients who had undergone surgical resection. A prognostic model that does not require pathologic variables needs to be developed and validated for patients who do not undergo surgical resection (12).

One of the crucial goals of risk stratification after detecting GBC in an incidentally resected gall bladder is to assess the likelihood of residual disease that dictates the decision of reresection and adjuvant therapy. Pathologic T-stage is the most reliable predictor of residual disease (4). The incidence of residual disease in patients with $\mathrm{T} 1$ tumors varies between
0 and $37.5 \%$, and extended re-resection is recommended in patients with $\mathrm{T} 1 \mathrm{~b}$ (invasion into the muscular layer) or higher $\mathrm{T}$ stage disease (4). A French collaborative group reported a superior 5-year OS in incidentally detected GBC patients $(\mathrm{n}=218)$ undergoing re-resection $(41 \%)$ compared to patients who did not undergo a re-resection (15\%) (14). In the study reported by Lohman et al., T1 tumors were not subclassified into T1a and T1b, limiting its applicability in patients with T1 tumors. Another major limitation of the study is that only $20.3 \%$ of patients underwent extended cholecystectomy, and the surgical procedures in $44.7 \%$ of patients were not specified. The lack of complete surgical staging in most patients limits the accuracy of the pathological staging, raising questions regarding the general applicability of this model. Additionally, it is important to note that $23.7 \%$ of patients in the development cohort had $\mathrm{R} 1$ resection that appears to be much higher than reported in other large studies, including the study reported by the French collaborative study group mentioned above in which only $3 \%$ of patients had R1 resection (14). These factors would influence the accuracy of the model.

Several other limitations are worth noting. The systemic therapies, including adjuvant chemotherapy and targeted therapies, can potentially influence the survival of patients with GBC (15). The study under discussion does not provide any information regarding systemic therapies. Moreover, the clinical data in the development cohort were collected retrospectively that may have compromised the quality of the data. In this study, tumor genomic sequencing data were not incorporated, which could have prognostic implications. Finally, the number of patients in the validation cohort was relatively small, as acknowledged by the authors in the discussion section of the paper. All these factors could adversely impact the accuracy of the model.

Despite the limitations discussed above, this study carries considerable clinical value as this is one of the largest studies reported based on nationwide, multi-institutional data in western patients. Additionally, the web application that emerged from this model will aid in treatment decisionmaking in routine clinical practice. It appears that paraffinembedded tissue blocks of these patients are accessible to the authors, which leads us to an expectation that authors will consider incorporating tumor genomic profiling data into this model in the future that will likely refine the predictive capability of this model.

\section{Acknowledgments}

Funding: None. 


\section{Footnote}

Provenance and Peer Review: This article was commissioned by the editorial office, Hepatobiliary Surgery and Nutrition. The article did not undergo external peer review.

Conflicts of Interest: Both authors have completed the ICMJE uniform disclosure form (available at https://hbsn. amegroups.com/article/view/10.21037/hbsn-21-514/coif). The authors have no conflicts of interest to declare.

Ethical Statement: The authors are accountable for all aspects of the work in ensuring that questions related to the accuracy or integrity of any part of the work are appropriately investigated and resolved.

Open Access Statement: This is an Open Access article distributed in accordance with the Creative Commons Attribution-NonCommercial-NoDerivs 4.0 International License (CC BY-NC-ND 4.0), which permits the noncommercial replication and distribution of the article with the strict proviso that no changes or edits are made and the original work is properly cited (including links to both the formal publication through the relevant DOI and the license). See: https://creativecommons.org/licenses/by-nc-nd/4.0/.

\section{References}

1. Lohman EAJS, de Bitter TJJ, Hannink G, et al. Development and External Validation of a Model to Predict Overall Survival in Patients with Resected Gallbladder Cancer. Ann Surg 2021. [Epub ahead of print].

2. Schmidt MA, Marcano-Bonilla L, Roberts LR. Gallbladder cancer: epidemiology and genetic risk associations. Chin Clin Oncol 2019;8:31.

3. Wistuba II, Gazdar AF. Gallbladder cancer: lessons from a rare tumour. Nat Rev Cancer 2004;4:695-706.

4. Zaidi MY, Abou-Alfa GK, Ethun CG, et al. Evaluation and management of incidental gallbladder cancer. Chin Clin Oncol 2019;8:37.

Cite this article as: Chakrabarti S, Mahipal A. Comment on: development and external validation of a model to predict overall survival in patients with resected gallbladder cancer. HepatoBiliary Surg Nutr 2022;11(1):136-138. doi: 10.21037/ hbsn-21-514
5. Giannis D, Cerullo M, Moris D, et al. Validation of the 8th Edition American Joint Commission on Cancer (AJCC) Gallbladder Cancer Staging System: Prognostic Discrimination and Identification of Key Predictive Factors. Cancers (Basel) 2021;13:547.

6. Oweira H, Mehrabi A, Giryes A, et al. External validation of the 8th American Joint Committee on Cancer staging system for gall bladder carcinoma. J Gastrointest Oncol 2018;9:1084-90.

7. Sung YN, Song M, Lee JH, et al. Validation of the 8th Edition of the American Joint Committee on Cancer Staging System for Gallbladder Cancer and Implications for the Follow-up of Patients without Node Dissection. Cancer Res Treat 2020;52:455-68.

8. Bai Y, Liu ZS, Xiong JP, et al. Nomogram to predict overall survival after gallbladder cancer resection in China. World J Gastroenterol 2018;24:5167-78.

9. Cai ZQ, Guo P, Si SB, et al. Analysis of prognostic factors for survival after surgery for gallbladder cancer based on a Bayesian network. Sci Rep 2017;7:293.

10. Chen C, Geng Z, Shen H, et al. Long-Term Outcomes and Prognostic Factors in Advanced Gallbladder Cancer: Focus on the Advanced T Stage. PLoS One 2016;11:e0166361.

11. Xiao Z, Shi Z, Hu L, et al. A new nomogram from the SEER database for predicting the prognosis of gallbladder cancer patients after surgery. Ann Transl Med 2019;7:738.

12. Yadav S, Tella SH, Kommalapati A, et al. A Novel Clinically Based Staging System for Gallbladder Cancer. J Natl Compr Canc Netw 2020;18:151-9.

13. Zhang W, Hong HJ, Chen YL. Establishment of a Gallbladder Cancer-Specific Survival Model to Predict Prognosis in Non-metastatic Gallbladder Cancer Patients After Surgical Resection. Dig Dis Sci 2018;63:2251-8.

14. Fuks D, Regimbeau JM, Le Treut YP, et al. Incidental gallbladder cancer by the AFC-GBC-2009 Study Group. World J Surg 2011;35:1887-97.

15. Kim TH, Woo SM, Lee WJ, et al. Benefit of Adjuvant Chemoradiotherapy in Resected Gallbladder Carcinoma. Sci Rep 2019;9:11770. 